\title{
Current mHealth Use in Dutch Dementia Care: A Guide to Action!
}

\author{
Thomas ENGELSMA ${ }^{\mathrm{a}, \mathrm{b}, 1}$, Ahsen YURT ${ }^{\mathrm{a}, \mathrm{b}}$, Monique W. JASPERS ${ }^{\mathrm{a}, \mathrm{b}}$, and Linda \\ DUSSELJEE-PEUTE ${ }^{\mathrm{a}, \mathrm{b}}$ \\ ${ }^{a}$ Center for Human Factors Engineering of Health Information Technology \\ ${ }^{\mathrm{b}}$ Department of Medical Informatics, Amsterdam Public Health research institute, \\ Amsterdam UMC, location AMC, Amsterdam, The Netherlands.
}

\begin{abstract}
Objective: To report underlying factors that hinder or advance mHealth implementation and use in Dutch dementia care. Methods: 44 dementia experts (healthcare professionals, informal caregivers, dementia case managers, and researchers) completed a questionnaire as part of a wider Delphi study to share their experiences with, thoughts on, and proposals for mHealth use in Dutch dementia care. A SWOT-framework is used to categorize the experiences, thoughts and proposals. Results: Four strengths, twelve weaknesses, thirteen opportunities and six threats for the current use of mHealth in Dutch dementia care were identified. Conclusion: Identified weaknesses highlight the importance for a guide for action for everyone involved in design and implementing mHealth for older adults with dementia. It is critical to raise awareness of mHealth's availability, improve its design, and continue to address the needs of older adults with dementia.
\end{abstract}

Keywords. mHealth, dementia, care, SWOT, COVID-19

\section{Introduction}

The number of available mHealth applications is ever-increasing as a result of individuals being more tech-savvy and the intensive use of smartphones, tablets, and other devices. In the field of dementia, mHealth applications are developed to address the needs and wishes of patients, (informal) caregivers, and health workers. Dementia care can utilize mHealth for cognitive training, activities of daily living, health and safety monitoring, leisure, socialization and navigation [1]. For (informal) caregivers, mHealth can provide resources, just-in-time information to solve problems, and stress reduction strategies [2]. Health workers that deliver primary healthcare services feel that mHealth leads to new ways of communication and relations with clients and throughout communities [3].

The coronavirus disease of 2019 (COVID-19) pandemic urged a digital revolution in dementia care [4]. Due to measures such as social distancing, research into technology to monitor older adults with dementia and improve distance communication increased [4]. In line with this demand, the purpose of this study is to report underlying factors that hinder or advance generic mHealth implementation and use in Dutch dementia care. This study is part of a larger Delphi study performed during the COVID-19 pandemic aiming

\footnotetext{
${ }^{1}$ Corresponding Author, Thomas Engelsma, Amsterdam UMC, University of Amsterdam, Department of Medical Informatics, Meibergdreef 9, Amsterdam, the Netherlands; E-mail: t.engelsma@amsterdamumc.nl
} 
to develop generic guidelines for the design of mHealth for older adults with dementia. A survey explored the participants' experiences with and thoughts on current mHealth use and proposals for future mHealth use in Dutch dementia care.

\section{Methods}

\subsection{Questionnaire development}

Data were gathered in a larger study on prioritizing potential barriers for older adults with dementia when using mHealth applications. In the introductory questionnaire of that study, demographic data and insights in mHealth experience were gathered from participants. Participants were informal caregivers, district and hospital nurses, geriatric specialists, researchers, and/or case managers and recruited (a) actively via email which led to snowball sampling as requests were forwarded to colleagues and (b) passively through organizations related to dementia care. The following four open-ended questions were asked: (1) What is your experience with the use of mHealth with elderly people with dementia, (2) What is your opinion on current mHealth applications for people with dementia, (3) Which barriers related to aging and dementia influence the use of mHealth, and (4) What are your suggestions for mHealth applications that can play a positive role for elderly people with dementia. The questions were pilot tested with eight dementia experts and was sent out using Castor EDC [5]. One question was added based on pilot feedback.

\subsection{SWOT-analysis}

The demographic questions were analyzed using descriptive statistics. The responses to the open-ended questions were categorized by TE and AY, using the SWOT framework, as either a Strength, Weakness, Opportunity, or Threat for mHealth use in Dutch dementia care. Afterwards, through thematic analysis, similar responses were grouped within each category of the framework by TE and discussed within the research team. To categorize the reported types of mHealth, responses were mapped on earlier defined mHealth types: perform daily activities, maintain social interaction, aid memory, engage in leisure activities, track location, and monitor health [6].

\section{Results}

The expert group $(n=44$, response rate $=81 \%)$ consisted of seventeen case managers, fifteen hospital or district nurses, one geriatrician, five researchers, and six informal caregivers. Most were female (90.9\%) and aged between 21-70. With respect to technology use, all participants used a smartphone and $66 \%$ reported having mHealth experience.

Participants indicated an increase in video calling on smartphones and tablets by older adults with dementia during the COVID-19 period as a medium for maintaining social interaction with family, friends, healthcare professionals and other (informal) caregivers. However, the older adults with dementia experienced barriers such as anxiety, agitation, and suspicion while video calling. During the COVID-19 pandemic, mHealth 
was also introduced to engage in leisure activities such as virtual museum visits and games which caused enthusiasm among users as activities during the pandemic were limited. Memory aid was already used mainly for reminding the older adults with dementia of upcoming events and to take their medications. Family members or informal caregivers already used tracking interventions to follow the location of older adults with dementia, and sensors to monitor health and behavior.

Four strengths, twelve weaknesses, thirteen opportunities and six threats were identified by the dementia experts and mentioned various times (Table 1).

Table 1. Strengths, weaknesses, opportunities and threats for the current use of mHealth in Dutch dementia care according to dementia experts (from most to least mentioned).

\begin{tabular}{lc}
\hline \multicolumn{1}{c}{ Strenghs } & $\#$ \\
\hline 1. Availability - To minimize social isolation, give daily structure, train memory, monitor health & 21 \\
circumstances, and facilitate distance communication, various mHealth applications can be offered & \\
based on the user's needs and wishes. & 11 \\
2. Support in early stages of dementia - When mHealth is introduced early in the disease's \\
progression, the user with dementia is or becomes comfortable with a smartphone or tablet and \\
when some form of roadmap is offered on how to utilize it, mHealth can provide support. \\
3. Enjoyment - Older adults with dementia seem to enjoy the use of mHealth. \\
4. Cost-effective - mHealth can be cost-effective for healthcare organizations. \\
\hline Weaknesses
\end{tabular}

1. Currently inaccessible - Current mHealth applications are for many people with dementia inaccessible, frequently overly complicated, and require a high level of cognitive ability.

2. Creates more burden for caregivers - People with dementia require support for using mHealth, which increases the burden of (informal) caregivers.

3. Resistant to use - Older adults with dementia can be resistant to mHealth due to (performance) anxiety or suspicion as the technology is unknown to them.

4. Difficult to set-up - Setting up mHealth requires too many actions (for example charging/updating).

5. Lack of awareness - Implementation and adoption is affected by the caregivers' or health workers' lack of awareness of or interest in mHealth.

6. Leads to frustration - Introducing mHealth to older adults with dementia is difficult and can lead to frustration.

7. Not appropriate for advanced stages - Healthcare workers often see people with further stages of dementia in which case the use of mHealth might not be appropriate.

8. Lack of evidence - There are many apps and tools to support dementia care, but an overview (what is available) and evidence for successful implementation is lacking.

9. Limited use in home care - mHealth use in home care settings is currently limited. in daily life, partially due to dementia.

11. Not meeting the needs - Current mHealth apps do not fully cover the needs for dementia care.

12. Organizational pressure - Organizational pressure to implement mHealth can lead to implementation issues and frustration for/of health workers and people with dementia.

\section{Opportunities}

1. Improve app design - Zooming in on personal qualities, motivations, and capacities of

(informal) caregivers and persons with dementia, as well as their influence on technology use, can improve mHealth design.

2. Focus on requirements - mHealth should require minimal actions, be provided on easy-to-use smartphones and remotely accessible by health workers or (informal) caregivers.

3. Teach (informal) caregivers - mHealth should be introduced early on in the course of dementia, and (informal) caregivers should be available and trained on its use.

4. Failure-free distraction - $\mathrm{mHea}$ th should be failure-free with positive feedback, provide pleasure and distraction, not confront dementia patients with their limitations, add to their quality of life, and be stimulus poor.

5. Focus on needs - People with dementia could gain from mHealth that provide games for memory training and support, reminisce, and reminders

6. Integrate in daily life - mHealth can be successful for people with dementia when it is integrated in their daily routine and provides reminders and support for daily activities, scheduling and signaling deviations in daily processes. 
7. Awaiting a more tech-savvy future - Future older adult generations will be more tech-savvy which may have a positive influence on tablet and smartphone use.

8. Create more awareness - The use of mHealth can be improved by raising awareness of the tools available to healthcare professionals

9. Counter problem behavior - $\mathrm{mHealth}$ can have value when it is used to counter problem behavior such as agitation in older adults with dementia.

10. More financial support - It is important to allocate more budget for mHealth initiatives and establish a robust business strategy to assure successful adoption of mHealth.

11. Adjust research focus - Research should not only focus on functional aspects of mHealth, but also on experiences with using technology.

12. Inclusion during implementation - People with dementia should be able to test and experience what mHealth has to offer and should not be left out of mHealth implementation processes.

13. Interoperability - mHealth should interoperate with current resources in dementia care.

\section{Threats}

1. Not owning a device - mHealth implementation is hindered when older adults with dementia or (informal) caregivers do not have interest in using or owning a smartphone, tablet or WiFi access

2. Stage and type of dementia - Health workers often visit older adults with more severe stages of dementia, which makes introducing mHealth and teaching them new skills more difficult

3. mHealth works temporarily - mHealth works temporarily due to apraxia, decreasing learnability and impaired cognitive function of dementia patients.

4. Demographics - Opportunities to use mHealth depend on demographic factors of dementia patients such as having a spouse or child(ren), financial situation, and living situation.

5. Physical limitations - Having a tremor or decreased fine motor skills hinders the smartphone or tablet use by patients with dementia.

6. Panic and mistrust - Decreased problem-solving abilities of dementia patients leads to panic and mistrust when mHealth fails.

\section{Discussion}

This paper discusses a subset of findings from a wider Delphi study identifying factors that influence generic mHealth implementation and use in Dutch dementia care. Apart from utilizing mHealth to prevent social isolation, provide daily structure, memory training and monitoring health conditions, dementia experts have seen an increase in mHealth use by dementia patients, healthcare providers and family caregivers to maintain social interaction (video calling) and to engage in leisure activities (such as virtual museum tours). This increase was a result of the COVID-19 restrictions, as older adults with dementia were not able to interact with family, friends and others involved in their care in person.

The findings show that there is still a need to raise awareness of mHealth's availability for dementia care. For example, some dementia experts see for example "providing memory support" as a strength of current mHealth use, while others saw this as an opportunity. However, the findings also imply that there is a growing interest in using mHealth among dementia experts, as they highlight particular scenarios, for example to counter problem behavior of their clients, in which situations they feel mHealth might be useful. Experts also suggest the implementation of remotely controlled mHealth, which they might have brought up as a result of COVID-19 restrictions. This rise coincides with a Norwegian study that found a 17 percent increase in caregivers' interest in technology to aid dementia care during the COVID-19 pandemic [7].

To enhance effective implementation and use of mHealth, the data emphasize to focus on user needs and requirements, provide failure-proof content, and personalize the design of mHealth. These findings are in line with those of a prior study in which 53 potential barriers to mHealth use for older adults with dementia were identified [8]. 
A limitation of this study is the exclusion of the intended user group, people with dementia, as inclusion of this group might have led to additional insights. In addition, thirty-four percent said they had no prior experience with mobile health. However, those participants might have been unaware of the wide range of technology that can be classified as mHealth.

We identified four strengths, twelve weaknesses, thirteen opportunities, and six threats based on the input of dementia experts, which can serve as a call to action for everyone involved in the development and implementation of mHealth for Dutch dementia care. Some responses were observed more frequently than others, but we recommend that all observed flaws and prospects for improving mHealth use in dementia care in the Netherlands should be critically evaluated.

\section{Conclusion}

mHealth can be utilized in early stages of dementia and might be cost-effective. Identified weaknesses highlight the importance for a guide for action for everyone involved in design and implementing mHealth for older adults with dementia. It is critical to raise knowledge of mHealth's availability among dementia experts, improve its design, and continue to address the needs of older adults with dementia.

\section{References}

[1] Yousaf K, Mehmood Z, Awan IA, Saba T, Alharbey R, Qadah T, Alrige MA. A comprehensive study of mobile-health based assistive technology for the healthcare of dementia and Alzheimer's disease (AD). Health Care Manag Sci. 2020 Jun;23(2):287-309.

[2] Grossman MR, Zak DK, Zelinski EM. Mobile Apps for Caregivers of Older Adults: Quantitative Content Analysis. JMIR Mhealth Uhealth. 2018 Jul 30;6(7):e162.

[3] Odendaal WA, Anstey Watkins J, Leon N, Goudge J, Griffiths F, Tomlinson M, Daniels K. Health workers' perceptions and experiences of using mHealth technologies to deliver primary healthcare services: a qualitative evidence synthesis. Cochrane Database Syst Rev. 2020 Mar 26;3(3):CD011942.

[4] Cuffaro L, Di Lorenzo F, Bonavita S, Tedeschi G, Leocani L, Lavorgna L. Dementia care and COVID-19 pandemic: a necessary digital revolution. Neurol Sci. 2020 Aug;41(8):1977-1979.

[5] Castor EDC. (2019). Castor Electronic Data Capture. [online] Available at: https://castoredc.com.

[6] Koo BM, Vizer LM. Examining Mobile Technologies to Support Older Adults With Dementia Through the Lens of Personhood and Human Needs: Scoping Review. JMIR Mhealth Uhealth. 2019 Nov $11 ; 7(11): \mathrm{e} 15122$.

[7] Gedde MH, Husebo BS, Erdal A, Puaschitz NG, Vislapuu M, Angeles RC, Berge LI. Access to and interest in assistive technology for home-dwelling people with dementia during the COVID-19 pandemic (PAN.DEM). Int Rev Psychiatry. 2021 Jan 8:1-8

[8] Engelsma T, Jaspers MWM, Peute LW. Considerate mHealth design for older adults with Alzheimer's disease and related dementias (ADRD): A scoping review on usability barriers and design suggestions. Int J Med Inform. 2021 Aug;152:104494. 